\title{
Long non-coding RNA HIF1A-AS1 is upregulated in intracranial aneurysms and participates in the regulation of proliferation of vascular smooth muscle cells by upregulating TGF- $\beta 1$
}

\author{
JIAMING XU ${ }^{1}$, YANG ZHANG ${ }^{1}$, LISHENG CHU $^{2}$, WEIYAN CHEN ${ }^{2}$, YUEGUANG DU $^{2}$ and JINGJING GU ${ }^{2}$ \\ ${ }^{1}$ Department of Neurosurgery, The Second Affiliated Hospital, Zhejiang University School of Medicine, \\ Hangzhou, Zhejiang 310000; ${ }^{2}$ Department of Pathology and Pathophysiology, School of Basic Medicine, \\ Zhejiang Chinese Medical University, Hangzhou, Zhejiang 310009, P.R. China
}

Received June 28, 2018; Accepted December 5, 2018

DOI: $10.3892 / \mathrm{etm} .2018 .7144$

\begin{abstract}
Long non-coding (lnc)RNA hypoxia inducible factor $1 \alpha$-antisense RNA 1 (HIF1A-AS1) not only participates in different types of malignancies, but also serves pivotal roles in thoracic aortic aneurysms, which suggests its possible involvement in intracranial aneurysms. Therefore, the present study aimed to investigate its involvement in intracranial aneurysms. Expression levels of HIF1A-AS1 and transforming growth factor (TGF)- $\beta 1$ in the blood of patients with intracranial aneurysms and healthy controls were detected using reverse transcription-quantitative polymerase chain reaction. The diagnostic value of blood HIF1A-AS1 for intracranial aneurysms was analyzed using receiver operating characteristic curve analysis. A HIF1A-AS1 expression vector was constructed and transfected into human vascular smooth muscle cells (VSMCs) and the effects on cell proliferation and TGF- $\beta 1$ expression were explored using the Cell Counting kit- 8 assay and western blot analysis, respectively. Upregulated HIF1A-AS1 expression levels in blood were observed in patients with intracranial aneurysms when compared with controls. Notably, upregulated HIF1A-AS1 expression effectively distinguished patients with intracranial aneurysms from healthy controls. Furthermore, HIF1A-AS1 and TGF- $\beta 1$ expression levels were positively correlated with intracranial aneurysms. HIF1A-AS1 overexpression also upregulated TGF- $\beta 1$ expression and inhibited VSMC proliferation. Although TGF- $\beta 1$ treatment had no significant effect on HIF1A-AS1
\end{abstract}

Correspondence to: Miss Jingjing Gu, Department of Pathology and Pathophysiology, School of Basic Medicine, Zhejiang Chinese Medical University, 548 Binwen Road, Hangzhou, Zhejiang 310009, P.R. China

E-mail: ytlfil5@163.com

Key words: intracranial aneurysm, hypoxia inducible factor $1 \alpha$-antisense RNA 1, transforming growth factor- $\beta 1$ expression, TGF- $\beta$ inhibitor significantly reduced the effects of HIF1A-AS1 overexpression on cell proliferation. It was therefore concluded that HIF1A-AS1 may participate in intracranial aneurysms by regulating VSMC proliferation through the upregulation of TGF- $\beta 1$.

\section{Introduction}

Intracranial aneurysms, also referred to as brain aneurysms, are pathological dilatations of the cerebral artery caused by weakness in the wall of a cerebral artery or vein (1). Intracranial aneurysms affect $3.2 \%$ of the whole population, and the numbers may increase in the near future due to the popularization of imaging techniques in the diagnosis $(2,3)$, which may increase the diagnosis rate of this disease. The existence of intracranial aneurysms may not significantly affect a patient's normal life; however, rupture of an intracranial aneurysm can cause life-threatening subarachnoid hemorrhages, which leads to high fatality and morbidity rates among those patients (4). At present, the treatment and prevention of intracranial aneurysms are still challenged due to their unknown pathogenesis.

Vascular smooth muscle cells (VSMCs) serve pivotal roles in maintaining vascular plasticity and the transition of VSMCs from normal conditions to matrix remodeling, and the pro-inflammatory phenotype is the primary pathological change in the development of an intracranial aneurysm (5). Transforming growth factor (TGF) $\beta \beta$ signaling is proved to participate in the regulation of VSMC proliferation (6), which indicates the potential involvement of TGF- $\beta$ signaling in the pathogenesis of intracranial aneurysm. TGF- $\beta$ signaling under certain conditions achieves its biological roles through interactions with different long non-coding (lnc)RNAs (7), which serve critical roles in human diseases (8). Recent studies have demonstrated that hypoxia inducible factor $1 \alpha$-antisense RNA 1 (HIF1A-AS1) may participate in the formation of thoracic aortic aneurysms by regulating the proliferation and apoptosis of VSMCs $(9,10)$. In the present study, the results suggested that HIF1A-AS1 may participate in the formation of intracranial aneurysms by upregulating TGF- $\beta 1$ and inhibiting VSMC proliferation. 


\section{Patients and methods}

Subjects and specimens. A total of 110 individuals were recruited to the current study. Blood specimens of 56 patients with intracranial aneurysms (30 males and 26 females; age range, 23-69 years old, with a mean age of $45.1 \pm 6.2$ years old) were obtained from the specimen library of Second Affiliated Hospital of Zhejiang University (Hangzhou, China). These patients were diagnosed and treated in Second Affiliated Hospital of Zhejiang University between January 2015 and January 2018. The inclusion criteria were as follows: Patients with complete medical records and willingness to be involved. The exclusion criteria were as follows: Patients complicated with other diseases or those that received any treatment (such as intravenous injection) prior to blood extraction were excluded from the present study. Blood samples were maintained at room temperature for $30 \mathrm{~min}$ and underwent centrifugation at $1,000 \times \mathrm{g}$ for $20 \mathrm{~min}$ at room temperature to collect serum (supernatant). At the same time, blood specimens of 54 healthy subjects (29 males and 25 females; age range, 24-67 years old, with a mean age of $44.7 \pm 5.9$ years old) who received routine physical examinations were also included to serve as the control group. There were significant differences between the groups with regard to age and sex. The ethic committee of Second Affiliated Hospital of Zhejiang University approved the present study and all participants provided their written, informed consent.

RNA extraction and reverse transcription-quantitative polymerase chain reaction (RT-qPCR). TRIzol reagent (Thermo Fisher Scientific., Inc., Waltham, MA, USA) was used to extract total RNA. All procedures were performed in strict accordance with the instructions of the kits used. cDNA was synthesized through RT using a SuperScript IV reverse transcriptase kit (Thermo Fisher Scientific, Inc.) with the following thermal conditions: $5 \mathrm{~min}$ at $25^{\circ} \mathrm{C}, 20 \mathrm{~min}$ at $52^{\circ} \mathrm{C}$ and $5 \mathrm{~min}$ at $80^{\circ} \mathrm{C}$. SYBR Green Real-Time PCR Master Mix (Thermo Fisher Scientific, Inc.) was used to prepare all reaction systems. The following primers were used in PCR reactions: Human IncRNA HIF1A-AS1 forward, 5'-AATGTGTTCCTTGCTCTT-3' and reverse, 5'-GTATGTCTCAGTTATCTTCCT-3'; and human GAPDH forward, 5'-CCCACTCCTCCACCTTTGAC-3' and reverse, 5'-ATGAGGTCCACCACCCTGTT-3'; TGF- $\beta 1$ mRNA primers were purchased from SinoBiological Inc. (Wayne, PA, USA; cat. no. HP100717). PCR reaction conditions were as follows: $1 \mathrm{~min}$ at $95^{\circ} \mathrm{C}$, followed by 40 cycles of $20 \mathrm{sec}$ at $95^{\circ} \mathrm{C}$ and $55 \mathrm{sec}$ at $62^{\circ} \mathrm{C}$. Data were normalized using the $2^{-\Delta \Delta \mathrm{Cq}}$ method (11).

Cell culture and transfection. Human VSMCs (cat. no. C0075C; Cascade Biologics; Thermo Fisher Scientific, Inc.) were cultured with Medium 231 containing smooth muscle growth supplements (both Cascade Biologics; Thermo Fisher Scientific, Inc.) in an incubator $\left(37^{\circ} \mathrm{C}, 5 \% \mathrm{CO}_{2}\right)$. An EcoRI-EcoRI fragment containing full HIF1A-AS1 cDNA was amplified through PCR and was inserted into pIRSE2 vector (Clontech Laboratories, Inc., Mountainview, CA, USA). Vectors were first mixed with Lipofectamine 2000 reagent (cat. no. 11668-019, Invitrogen; Thermo Fisher Scientific, Inc.) to construct vector-reagent complexes. Following this, cells were incubated at $37^{\circ} \mathrm{C}$ in an atmosphere containing $5 \% \mathrm{CO}_{2}$ for $5 \mathrm{~h}$. Subsequently, cells were immediately washed with fresh culture medium to avoid cytotoxicity. Cells without transfection were used as control cells (C). Cells transfected with empty vector were used as negative control cells (NC). In the analysis of the effects of HIF1A-AS1 on TGF- $\beta 1$, cells were treated with exogenous TGF- $\beta 1$ (Sigma-Aldrich; Merck KGaA, Darmstadt, Germany) at 10 and $20 \mathrm{ng} / \mathrm{ml}$ for 6,12 and $18 \mathrm{~h}$ prior to use. In the analysis of the effects of HIF1A-AS1 overexpression and TGF- $\beta 1$ signaling on VSMC proliferation, cells were treated with TGF- $\beta 1(10 \mathrm{ng} / \mathrm{ml})$ for $12 \mathrm{~h}$ or TGF- $\beta 1$ inhibitor LY-364947 (LY, cat. no. 616451; Merck KGaA) for $12 \mathrm{~h}$ prior to use. Cells were harvested $24 \mathrm{~h}$ after transfection for subsequent experiments.

Cell proliferation assay. Following transfection and confirmation of overexpression (overexpression rate, $<200 \%$ ), cells were collected to prepare cell suspensions using a cell density of $4 \times 10^{4}$ cells per $1 \mathrm{ml}$. Cell suspension $(100 \mu \mathrm{l})$ was added into each well of a 96-well plate. The plate was incubated in an incubator $\left(37^{\circ} \mathrm{C}, 5 \% \mathrm{CO}_{2}\right)$. Subsequently, $10 \mu \mathrm{l}$ CCK-8 solution (Sigma-Aldrich; Merck KGaA) was added into each well at $24,48,72$ and $96 \mathrm{~h}$ following the beginning of cell culture. Cells were cultured in an incubator $\left(37^{\circ} \mathrm{C}, 5 \% \mathrm{CO}_{2}\right)$ for a further $4 \mathrm{~h}$. A Fisherbrand accuSkan GO UV/Vis Microplate Spectrophotometer (Thermo Fisher Scientific, Inc.) was used to measure the optical density values at $450 \mathrm{~nm}$. Cell proliferation was normalized to C $(100 \%)$.

Western blot analysis. Radioimmunoprecipitation assay lysis solution (Thermo Fisher Scientific, Inc.) was used to extract total protein from in vitro cultured cells transfected with HIF1A-AS1 expression vectors, control cells and negative control cells, according to the manufacturer's instructions. Protein concentrations were measured using a BCA assay. Protein samples were mixed with $6 \mathrm{X}$ loading buffer with a ratio of 1:5. Following denaturing at $95^{\circ} \mathrm{C}$, SDS-PAGE (10\% gels) was performed with $30 \mu \mathrm{g}$ protein loaded per lane. Proteins were transferred on polyvinylidene difluoride (PVDF) membranes (Thermo Fisher Scientific, Inc.) and blocked in $5 \%$ skimmed milk at room temperature for $2 \mathrm{~h}$. Membranes were incubated with rabbit anti-human primary antibodies of TGF- $\beta 1$ (1:2,000; cat. no. ab92486) and GAPDH antibody (1:2,000; cat. no. ab181602; both Abcam, Cambridge, MA, USA) at $4^{\circ} \mathrm{C}$ overnight. The following day, PVDF membranes were incubated with goat anti-rabbit IgG-horseradish peroxidase secondary antibody (1:1,000; cat. no. MBS435036; MyBioSource, Inc., San Diego, CA, USA) at room temperature for $2 \mathrm{~h}$. Pierce ECL Western Blotting Substrate (Thermo Fisher Scientific., Inc.) was then dropped onto membranes to develop signals. Signal normalization was performed using Image 1.48 software (National Institutes of Health, Bethesda, MD, USA).

Statistical analysis. GraphPad Prism 6 software (GraphPad Software, Inc., La Jolla, CA, USA) was used for all statistical analyses. All data were recorded as the mean \pm standard deviation and the unpaired t-test and one-way analysis of variance followed by the Least Significant Difference post hoc test were used for comparisons between two groups and among multiple groups, respectively. Correlation analyses were performed 


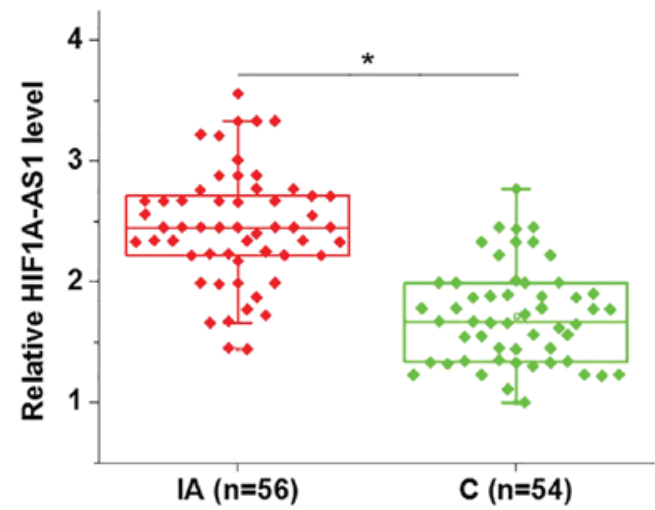

Figure 1. Blood levels of long non-coding RNA HIF1A-AS1 are upregulated in IAs. ${ }^{*} \mathrm{P}<0.05$ as indicated. IA, intracranial aneurysm; $\mathrm{C}$, healthy control; HIF1A-AS1, hypoxia inducible factor $1 \alpha$-antisense RNA 1.

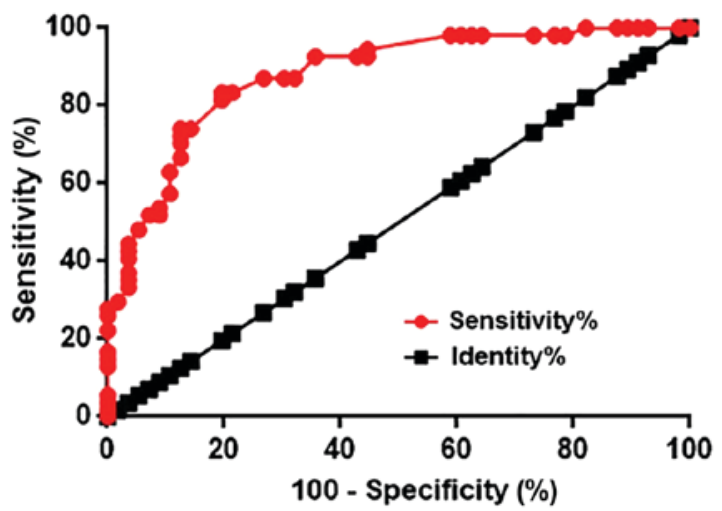

Figure 2. Receiver operating characteristic curve analysis of the diagnostic value of blood hypoxia inducible factor $1 \alpha$-antisense RNA 1 for intracranial aneurysms.
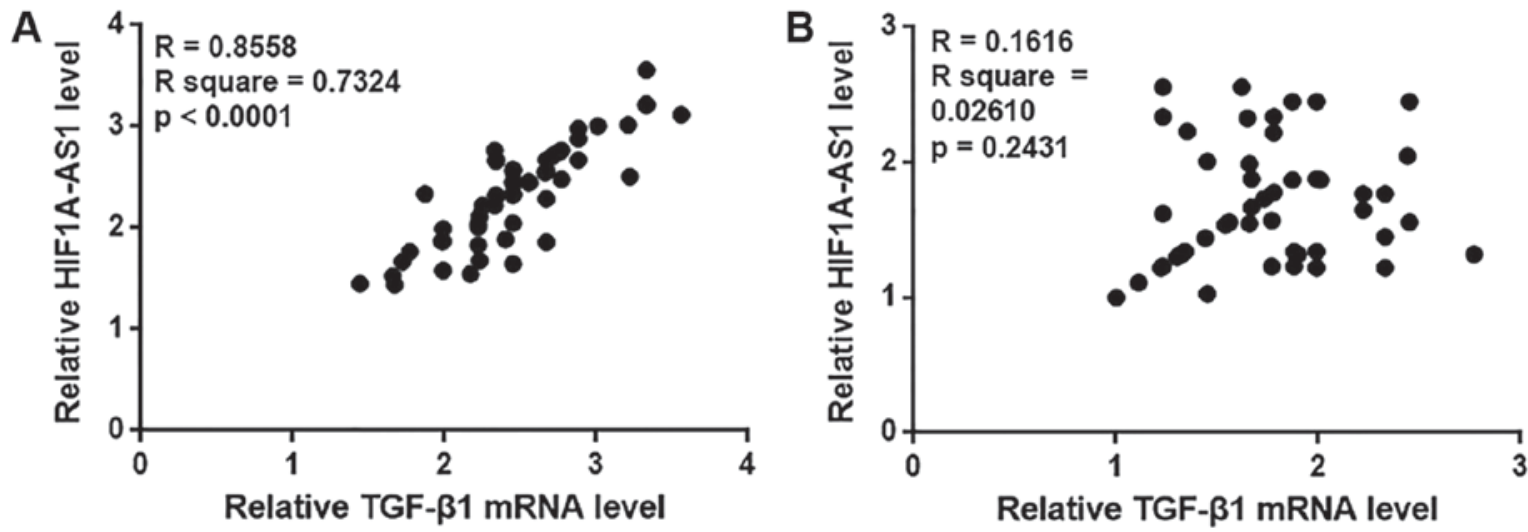

Figure 3. HIF1A-AS1 and TGF- $\beta 1$ expression are positively correlated in patients with intracranial aneurysms but not in healthy controls. Pearson correlation coefficient analysis was performed to assess the correlation between HIF1A-AS1 and TGF- $\beta 1$ expression in the blood of patients with (A) intracranial aneurysm and (B) healthy controls. HIF1A-AS1, hypoxia inducible factor $1 \alpha$-antisense RNA 1 ; TGF, transforming growth factor.

using Pearson correlation coefficient. $\mathrm{P}<0.05$ was considered to indicate a statistically significant difference.

\section{Results}

Expression levels of IncRNA HIFIA-AS1 in the blood are upregulated in patients with intracranial aneurysms. In the present study, the expression levels of lncRNA HIF1A-AS1 in the blood of patients with intracranial aneurysms and healthy controls were detected by RT-qPCR. As indicated in Fig. 1, the expression levels of HIF1A-AS1 were identified to be significantly increased in patients with intracranial aneurysms compared with healthy controls.

LncRNA HIF1A-AS1 in the blood may serve as a potential biomarker for intracranial aneurysms. Receiver operating characteristic (ROC) curve analysis was performed to evaluate the diagnostic value of HIF1A-AS1 in the blood for intracranial aneurysms. As indicated in Fig. 2, the area under the curve was 0.8798 , with a standard error of 0.03213 and a 95\% confident interval of 0.8168-0.9428. Therefore, upregulation of HIF1A-AS1 in the blood effectively distinguished patients with intracranial aneurysms from healthy controls.
HIF1A-AS1 and TGF- $\beta 1$ expression levels are positively correlated in patients with intracranial aneurysms but not in healthy controls. In the present study, the Pearson correlation coefficient was used to analyze the correlation between HIF1A-AS1 and TGF- $\beta 1$ expression in the blood. As demonstrated in Fig. 3A, a significant positive correlation was identified between HIF1A-AS1 and TGF- $\beta 1$ expression in the blood of patients with intracranial aneurysms. By contrast, no significant correlation between HIF1A-AS1 and TGF- $\beta 1$ expression was observed in the blood of healthy controls (Fig. 3B).

HIF1A-AS1 is likely an upstream activator of TGF- $\beta 1$ in human VSMCs. To further explore the interactions between HIF1A-AS1 and TGF- $\beta 1$, HIF1A-AS1 expression vector was transfected in to VSMCs and the expression levels of TGF- $\beta 1$ were detected by western blot analysis. As revealed in Fig. 4A, VSMC cells transfected with HIF1A-AS1 expression vectors (HIF1A-AS1) exhibited significantly upregulated expression levels of TGF- $\beta 1$ protein compared with $\mathrm{C}$ and $\mathrm{NC}$. By contrast, cells treated with exogenous TGF- $\beta 1$ for 6,12 and $18 \mathrm{~h}$ exhibited no significant changes in HIF1A-AS1 expression (Fig. 4B).

HIF1A-AS1 overexpression inhibits the proliferation of VSMCs. The CCK-8 assay was performed to detect VSMC proliferation 
A

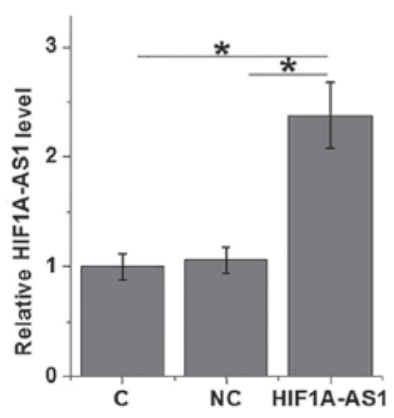

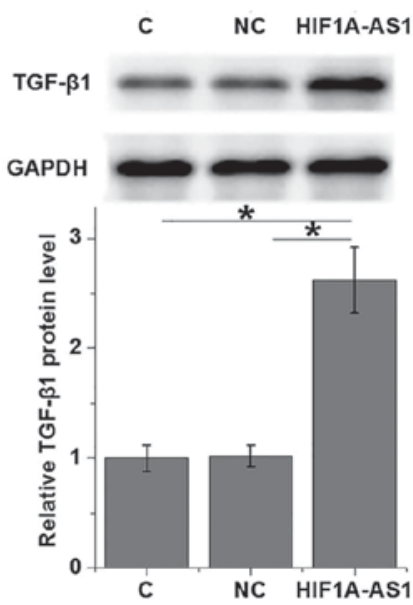

B

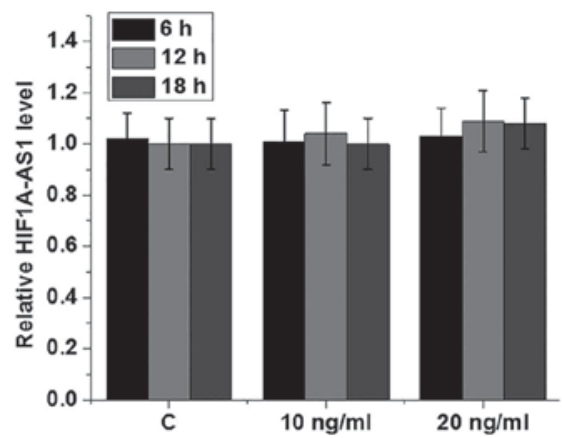

Figure 4. HIF1A-AS1 is likely an upstream activator of TGF- $\beta 1$ in human VSMCs. (A) HIF1A-AS1 overexpression was successfully induced. In addition, the effects of HIF1A-AS1 overexpression on TGF- $\beta 1$ expression and (B) the effects of exogenous TGF- $\beta 1 \mathrm{treatment}(10 \mathrm{ng} / \mathrm{ml}$ or $20 \mathrm{ng} / \mathrm{ml})$ on HIF1A-AS1 expression in human VSMCs were indicated. * $\mathrm{P}<0.05$ as indicated. HIF1A-AS1, hypoxia inducible factor $1 \alpha$-antisense RNA 1 ; TGF, transforming growth factor; VSMC, vascular smooth muscle cell; $\mathrm{C}$, control cells; $\mathrm{NC}$, negative control cells.

following HIF1A-AS1 overexpression; the rate of overexpression was $>200 \%$ compared with control cells (data not shown). As indicated in Fig. 5, compared with C, VSMC proliferation was significantly inhibited when cells were transfected with HIF1A-AS1 expression vector and $10 \mathrm{ng} / \mathrm{ml} \mathrm{TGF-} \beta 1$. In addition, compared with VSMCs treated with HIF1A-AS expression vector only, cells treated with HIF1A-AS expression vector transfection and the TGF- $\beta 1$ inhibitor LY exhibited significantly increased cell proliferation.

\section{Discussion}

The present study reported that HIF1A-AS1 was involved in in the pathogenesis of intracranial aneurysms. Notably, the present study indicated that HIF1A-AS1 expression inhibited VSMC proliferation and upregulated TGF- $\beta 1$ expression.

Although the pathogenesis of aneurysms is still unclear, previous research has indicated that the development and progression of different types of aneurysms are usually accompanied with changes in expression of a large set of non-coding RNAs (12). In a recent study, Wang et al (13) reported 4,129 differentially expressed lncRNAs between intracranial aneurysm tissues and superficial temporal arteries, and those lncRNAs were identified to likely be involved in intracranial aneurysms through their roles in the regulating the immune and inflammatory response. Upregulation of HIF1A-AS1 has been observed in thoracic aortic aneurysms (9). In the present study, significantly increased blood levels of HIF1A-AS1 in patients with intracranial aneurysms were observed compared with in heathy controls, indicating that upregulation of HIF1A-AS1 may also participate in the pathogenesis of intracranial aneurysms.

Currently, the diagnosis of intracranial aneurysms predominantly relies on imaging techniques, such as computerized tomography (14) and magnetic resonance imaging (15). However, only $\sim 10 \%$ of patients with intracranial aneurysms present with clinical symptoms before rupture (16), and early diagnosis, which is critical for survival, is not common. The onset, development and progression of human disease are usually accompanied with changes in certain blood substances,

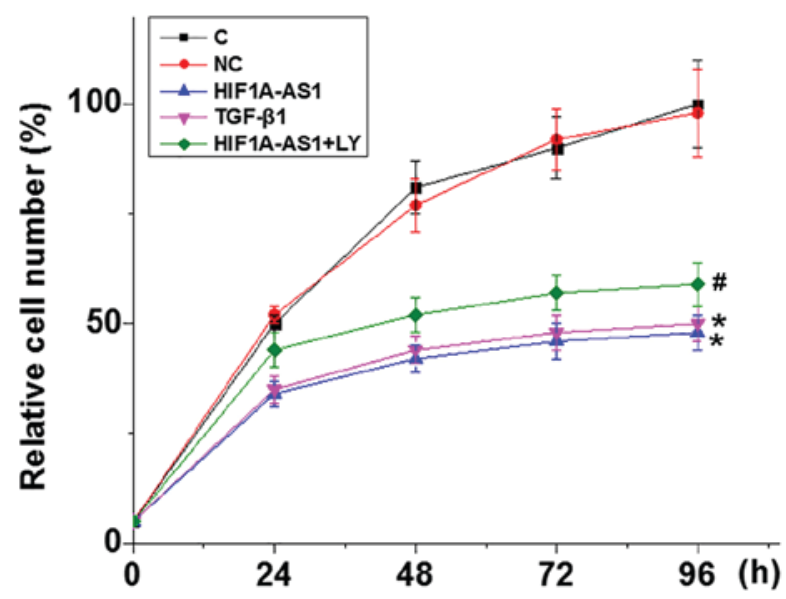

Figure 5. HIF1A-AS1 overexpression inhibits the proliferation of vascular smooth muscle cells. ${ }^{*} \mathrm{P}<0.05$ vs. C. ${ }^{\#} \mathrm{P}<0.05$ vs. HIF1A-AS1. HIF1A-AS1, hypoxia inducible factor $1 \alpha$-antisense RNA 1 ; TGF, transforming growth factor; C, control cells; NC, negative control cells.

and monitoring changes in those blood substances may provide guidance for disease diagnosis (17). In the present study, blood HIF1A-AS1 was detected in all patients with intracranial aneurysms and healthy controls. ROC curve analysis indicated that blood HIF1A-AS1 can be used to effectively distinguish patients with intracranial aneurysms from healthy controls. Therefore, blood HIF1A-AS1 may serve as a potential diagnostic marker for intracranial aneurysms. However, altered expression of HIF1A-AS1 has been observed in different diseases, such as thoracic aortic aneurysms (9) and non-small cell lung cancer (18). Therefore, multiple markers should be used to improve the diagnostic specificity.

The present study observed a significant correlation between HIF1A-AS1 and TGF- $\beta 1$ expression in patients with intracranial aneurysms but not in healthy controls, indicating the existing of an intracranial aneurysm-specific interaction between HIF1A-AS1 and TGF- $\beta 1$. HIF1A-AS1 is likely an upstream activator of TGF- $\beta 1$ in VSMCs due to the following reasons: 
i) HIF1A-AS1 overexpression promoted TGF- $\beta 1$; ii) exogenous TGF- $\beta 1$ produced no significant effects on HIF1A-AS1 expression; and iii) exogenous TGF- $\beta 1$ inhibitor reduced the effects of HIF1A-AS1 overexpression in VSMCs proliferation. It is worth noting that a recent study reported that the TGF- $\beta$ signaling pathway promotes VSMCs proliferation (19), whereas the present study revealed that TGF- $\beta 1$ inhibited the proliferation of VSMCs. The inconsistency between the present data and this previous study is possibly due to the different VSMCs used in the present study

In conclusion, the present study suggested that HIF1A-AS1 is upregulated in patients with intracranial aneurysms. The findings indicate that HIF1A-AS1 may participate in the pathogenesis of HIF1A-AS1 by upregulating TGF- $\beta 1$ and inhibiting VSMCs proliferation. However, the current study is limited by the small sample size. Future studies with bigger sample size are required to further confirm the conclusions of the current study.

\section{Acknowledgements}

Not applicable.

\section{Funding}

The present study was funded by the National Natural Science Foundation of China (grant nos. 81501016 and 81603487), the Natural Science Foundation of Zhejiang Province (grant no. LY14H09006) and the Science and Technology Project of Zhejiang Province (grant no. 2014F81G2010024).

\section{Availability of data and materials}

The analyzed data sets generated during the study are available from the corresponding author on reasonable request.

\section{Authors' contributions}

JX and JG designed experiments. JX, YZ and LC performed experiments. WC and YD prepared materials and analyzed the data. JG interpreted data and drafted the experiment. All authors approved the manuscript.

\section{Ethics approval and consent to participate}

The ethic committee of Zhejiang Chinese Medical University Hospital approved the present study and all participants provided their written, informed consent.

\section{Patient consent for publication}

Not applicable.

\section{Competing interests}

The authors declare that they have no competing interests.

\section{References}

1. Cebral JR, Duan X, Chung BJ,Putman C, Aziz K and Robertson AM: Wall mechanical properties and hemodynamics of unruptured intracranial aneurysms. AJNR Am J Neuroradiol 36: 1695-1703, 2015.
2. Anderson JR, Thompson WL, Alkattan AK, Diaz O, Klucznik R, Zhang YJ, Britz GW, Grossman RG and Karmonik C: Three-dimensional printing of anatomically accurate, patient specific intracranial aneurysm models. J Neurointerv Surg 8: 517-520, 2016.

3. Vlak MH, Algra A, Brandenburg R and Rinkel GJ: Prevalence of unruptured intracranial aneurysms, with emphasis on sex, age, comorbidity, country, and time period: A systematic review and meta-analysis. Lancet Neurol 10: 626-636, 2011.

4. Korja M and Kaprio J: Controversies in epidemiology of intracranial aneurysms and SAH. Nat Rev Neurol 12: 50-55, 2016.

5. Starke RM, Chalouhi N, Ding D, Raper DM, Mckisic MS, Owens GK, Hasan DM, Medel R and Dumont AS: Vascular smooth muscle cells in cerebral aneurysm pathogenesis. Transl Stroke Res 5: 338-346, 2014.

6. Tsai S, Hollenbeck ST, Ryer EJ, Edlin R, Yamanouchi D, Kundi R, Wang C, Liu B and Kent KC: TGF- $\beta$ through Smad3 signaling stimulates vascular smooth muscle cell proliferation and neointimal formation. American Journal of Physiology-Heart and Circulatory Physiology 297: H540-H549, 2009.

7. Tu X, Zhang Y, Zheng X, Deng J, Li H, Kang Z, Cao Z, Huang Z, Ding Z, Dong L, et al: TGF- $\beta$-induced hepatocyte lincRNA-p21 contributes to liver fibrosis in mice. Sci Rep 7: 2957, 2017.

8. Shi X, Sun M, Liu H, Yao Y and Song Y: Long non-coding RNAs: A new frontier in the study of human diseases. Cancer Lett 339: 159-166, 2013.

9. Zhao Y, Feng G, Wang Y, Yue Y and Zhao W: Regulation of apoptosis by long non-coding RNA HIF1A-AS1 in VSMCs: implications for TAA pathogenesis. Int J Clin Exp Pathol 7: 7643-7652, 2014.

10. Wang S, Zhang X, Yuan Y, Tan M, Zhang L, Xue X, Yan Y, Han L and Xu Z: BRG1 expression is increased in thoracic aortic aneurysms and regulates proliferation and apoptosis of vascular smooth muscle cells through the long non-coding RNA HIF1A-AS1 in vitro. Eur J Cardiothorac Surg 47: 439-446, 2015.

11. Livak KJ and Schmittgen TD: Analysis of relative gene expression data using real-time quantitative PCR and the 2(-Delta Delta (CT)) method. Methods 25: 402-408, 2001.

12. Duggirala A, Delogu F, Angelini TG, Smith T, Caputo M, Rajakaruna C and Emanueli C: Non coding RNAs in aortic aneurysmal disease. Front Genet 6: 125, 2015.

13. Wang W, Li H, Yu L, Zhao Z, Wang H, Zhang D, Zhang Y, Lan Q, Wang J and Zhao J: Aberrant expression of lncRNAs and mRNAs in patients with intracranial aneurysm. Oncotarget 8: 2477-2484, 2017.

14. Shintai K, Matsubara N and Izumi T: High-resolution cone beam CT for evaluation of vascular channel in intracranial partial thrombosed aneurysm. Nagoya J Med Sci 80: 279-284, 2018.

15. Vakil P, Ansari SA, Cantrell CG, Eddleman CS, Dehkordi FH, Vranic J, Hurley MC, Batjer HH, Bendok BR and Carroll TJ: Quantifying intracranial aneurysm wall permeability for risk assessment using dynamic contrast-enhanced MRI: A pilot study. AJNR Am J Neuroradiol 36: 953-959, 2015.

16. Wiebers DO, Whisnant JP, Huston J III, Meissner I, Brown RD Jr, Piepgras DG, Forbes GS, Thielen K, Nichols D, O'Fallon WM, et al: International study of unruptured intracranial aneurysms investigators. Unruptured intracranial aneurysms: Natural history, clinical outcome, and risks of surgical and endovascular treatment. Lancet 362: 103-110, 2003.

17. De Sanctis P, Elmakky A, Farina A, Caramelli E, Seracchioli R, Mabrouk M, Mignemi G, Venturoli S, Villa G, Guerrini M, et al: Matrix metalloproteinase-3 mRNA: A promising peripheral blood marker for diagnosis of endometriosis. Gynecol Obstet Invest 71: 118-123, 2011.

18. Tantai J, Hu D, Yang Y and Geng J: Combined identification of long non-coding RNA XIST and HIF1A-AS1 in serum as an effective screening for non-small cell lung cancer. Int J Clin Exp Pathol 8: 7887-7895, 2015.

19. DiRenzo DM, Chaudhary MA, Shi X, Franco SR, Zent J, Wang K, Guo LW and Kent KC: A crosstalk between TGF- $\beta /$ Smad 3 and Wnt $/ \beta$-catenin pathways promotes vascular smooth muscle cell proliferation. Cell Signal 28: 498-505, 2016.

This work is licensed under a Creative Commons Attribution-NonCommercial-NoDerivatives 4.0 International (CC BY-NC-ND 4.0) License. 